\title{
First case of B ALL with KMT2A-MAML2 rearrangement: a case report
}

\author{
Estelle Menu ${ }^{{ }^{*}}$, Nathalie Beaufils ${ }^{1}$, Fabrice Usseglio ${ }^{1,2}$, Estelle Balducci ${ }^{3}$, Marina Lafage Pochitaloff ${ }^{3}$, \\ Regis Costello ${ }^{4}$ and Jean Gabert ${ }^{1,2}$
}

\begin{abstract}
Background: A large number of chromosomal translocations of the human KMT2A gene, better known as the MLL gene, have so far been characterized. Genetic rearrangements involving KMT2A gene are frequently involved in lymphoid, myeloid and mixed lineage leukemia. One of its rare fusion partners, the mastermind like 2 (MAML2) gene has been reported in four cases of myeloid neoplasms after chemotherapy so far: two acute myeloid leukemias (AML) and two myelodysplasic syndrome (MDS), and two cases of secondary T-cell acute lymphoblastic leukemia (T-ALL).

Case presentation: Here we report the case of a KMT2A - MAML2 fusion discovered by Next-Generation Sequencing (NGS) analysis in front of an inv11 (q21q23) present in a 47-year-old female previously treated for a sarcoma in 2014, who had a B acute lymphoid leukemia (B ALL).

Conclusion: It is, to our knowledge, the first case of B acute lymphoblastic leukemia with this fusion gene. At the molecular level, two rearrangements were detected using RNA sequencing juxtaposing exon 7 to exon 2 and exon 9 to intron 1-2 of the KMT2A and MAML2 genes respectively, and one rearrangement using Sanger sequencing juxtaposing exon 8 and exon 2.
\end{abstract}

Keywords: Secondary ALL, KMT2A rearrangement, MAML2 gene, Next-Generation Sequencing

\section{Background}

Over $70 K M T 2 A$ partner genes have been reported so far in the literature. Translocation of the KMT2A gene, better known as the $M L L$ gene, is present in $7-10 \%$ of $\mathrm{B}$ acute lymphoblastic leukemias, AFF1 (or AF4) being the most frequent partner gene. The prognosis of $B$ acute lymphoblastic leukemias (B ALL) with $K M T 2 A$ gene translocations are usually poor, especially with $K M T 2 A$ AFF1 fusions [1, 2]. One of its rare partner genes, mastermind like 2 (MAML2) gene has been reported in four cases of myeloid neoplasms after chemotherapies: two cases of acute myeloid leukemia (AML) and two cases of myelodysplasic syndromes (MDS) [3]. Secondary acute lymphoid leukemia is a well-documented occurrence following topoisomerase II inhibitors and cyclophosphamide chemotherapy and/or radiotherapy. The premise that radiotherapy as well as chemotherapy are inducing $\mathrm{t}(4 ; 11)(\mathrm{q} 21 ; \mathrm{q} 23)$ was raised before [4].

\footnotetext{
* Correspondence: Estelle.MENU@ap-hm.fr

'Department of Biochemistry \& Molecular Biology, University Hospital Nord, Marseille, France

Full list of author information is available at the end of the article
}

Indeed two cases of secondary T-cell acute lymphoblastic leukemia with $K M T 2 A-M A M L 2$ transcripts have been described in two adolescent leukemia patients [5].

At the molecular level, much progress has been made in the identification of fusion transcripts and finding new alternatives to fluorescent in situ hybridization (FISH). In fact, the identification of fusion transcripts is possible with the Next-Generation Sequencing (NGS) technologies, such as RNA-Sequencing [6]. At the moment, this method seems to be the best for identifying new gene fusion transcripts, and is steadily improving [7]. In this case, we highlight one of the limitations of this powerful tool.

\section{Case presentation}

Patient's history and clinical features

A 47-year-old female, with morbid obesity and hypertrophic cardiopathy, presented to hospital in cardiopulmonary arrest most likely due to a cardiac rhythm disturbance. During hospitalization in the cardiac section, laboratory analysis revealed anemia ( $\mathrm{Hb} 105 \mathrm{~g} / \mathrm{L})$, thrombocytopenia $\left(59 \times 10^{9}\right.$ platelets/L) and a total leukocyte count of $6.2 \times 10^{9} / \mathrm{L}$ with $1 \%$ blast cells. Bone 
marrow aspiration showed hyper cellular marrow with agranular leukemic cells (35\%). The examination of the bone marrow morphology revealed dysplasia on the three cell lineages. Flow cytometric immunophenotyping of bone marrow identified blast cells that were positive for cCD79a, CD22, CD19 and CD45, and negative for CD10. There was a history of high-grade thoracic sarcoma in the previous year, treated with surgical resection, three cycles of chemotherapy (Doxorubicin/ Ifosfamide) and then radiotherapy. This patient had several thrombocytopenic episodes and encephalopathy due to ifosfamide. Hence, the patient was diagnosed with $\mathrm{B}$ ALL with a very poor prognosis secondary to the sarcoma treatment. Induction chemotherapy with cytarabine and anthracycline was started, but only one cycle of chemotherapy was achieved due to general deterioration, leading to an admission to intensive care. The patient deteriorated rapidly and passed away.

\section{Patient's molecular features}

Bone marrow conventional cytogenetic analysis showed a complex hyperdiploid karyotype with multiple structural and numerical abnormalities including 2p11 deletion and duplication of the inverted chromosome 11. Based on R-banding analysis, the karyotype was interpreted as 63, XX, $+\operatorname{del}(2)(\mathrm{p} 11),+5,+6,+7,+8,+9,+9$, $+10,+10,+11,+12,+13,+14,+15,+19,+20,+22[22] / 46, \quad \mathrm{XX}$ [4] (Fig. 1c). FISH analysis was performed on bone marrow aspirate (Fig. 1a) using a LSI KMT2A break apart probe (Vysis, Abbott Molecular Inc.). It revealed the presence of one normal chromosome and two inverted 11 chromosomes, each carrying a KMT2A gene rearrangement with an unknown fusion partner, in four metaphases and in 58/100 nuclei (Fig. 1b).

RNA sequencing (RNA-Seq) has been used to identify gene fusions on RNA extracted from the patient's blood. Venous blood $(10 \mathrm{~mL})$ was collected in EDTA tubes from the patient and PBMC were isolated by erythrocytes lysis (Buffer EL, Qiagen). Total RNA was extracted from $1 \times 10^{7}$ cells using Trizol Reagent (life technologies). For paired-end RNA-Seq, we have used the TruSeq RNA Library Prep Kits followed by paired-end cluster generation and sequencing using the $2 \times 150$ NextSeq 500 high output kit and the Illumina NextSeq 500 sequencing platform. The first variant (Var 1) corresponded to the fusion of the exon 7 of $K M T 2 A$ and the exon 2 of $M A M L 2$ as described previously [3]. For the second variant ( $\operatorname{Var} 2$ ), the data revealed that the fusion point was within the exon 9 of KMT2A gene and within the intron 1-2 of $M A M L 2$ gene. To validate these RNASeq results, we have performed RT-PCR with specific primers localized on exon 7 or exon 8 of KMT2A gene and intron 1-2 or exon 2 of $M A M L 2$ gene and Sanger sequencing (Fig. 2). The results had confirmed the presence of the two variants but had showed unexpectedly a new variant (Var 3). This fusion gene corresponded to the rearrangement juxtaposing exon 8 and exon 2 of the $K M T 2 A$ and $M A M L 2$ genes respectively and was detected with a higher expression level than the other variants (Fig. 3).

\section{Discussion and conclusions}

Here, we reported the first case of a B ALL in a 47 years old woman bearing a KMT2A-MAML2 rearrangement detected by NGS RNA analysis. As a result, KMT2A$M A M L 2$ rearrangement can be found in several types of acute leukemia. Moreover, we have detected two new variants of this fusion transcript based on exon fusion analysis. In fact, RNA sequencing juxtaposing exons 9 of the $K M T 2 A$ to intron $1-2$ of the $M A M L 2$ gene, and exon 8 of the $K M T 2 A$ to exon 2 of the $M A M L 2$ gene, have not previously been reported.

Three hypotheses may explain these molecular findings. Either three different breakpoints exist, or/and it is the result of alternative splicing. Indeed, mRNA precursors could lead to the production, via alternative splicing, of a fusion transcript exon 8 of the $K M T 2 A$ gene to exon 2 of the $M A M L 2$ gene and exon 7 of the KMT2A gene to exon 2 of the $M A M L 2$ gene. Alternative splicing are mechanisms invoked to play an important role in influencing the mutation effect [8]. The possibility of exons' skipping or inclusion are almost unlimited, thus, any gene could potentially lead to tens or hundreds of distinctive transcripts. Moreover, we ruled out the possibility of a pre-mRNA by RNA sequencing and PCR. In fact, pre-mRNA contains both introns and exons, and requires splicing of introns to produce the final mRNA molecule containing only exons. In our case, after RNA sequencing and PCR only intron 1-2 of the $M A M L 2$ gene persists and this variant was found in significant quantity. Each of the putative fusion transcripts was then translated into predicted amino acid sequences and each of the putative fusion proteins characterized. Two of the three variants (Var 2 and Var 3) contained in-frame stops and are unlikely to encode functional proteins. For the third variant (Var 1), the transcript contained the boundary exons and generated putative fusion transcript.

Furthermore, this case highlights one of the limitations of NGS. In fact, two different KMT2A-MAML2 gene fusions were found by NGS technology, but the major fusion variant, juxtaposing exons 8 of the KMT2A gene and exon 2 of the $M A M L 2$ gene, was not detected. NGS methods have the ability to detect a wide array of mutation types, which is mostly limited by the need to know all potential off-target capture regions and to properly process the raw data generated by the sequencer [9]. We had to use Sanger sequencing with specific primers to detect this third variant of KMT2A-MAML2 gene fusion, which was clearly demonstrated on the PCR gel (Fig. 2). 

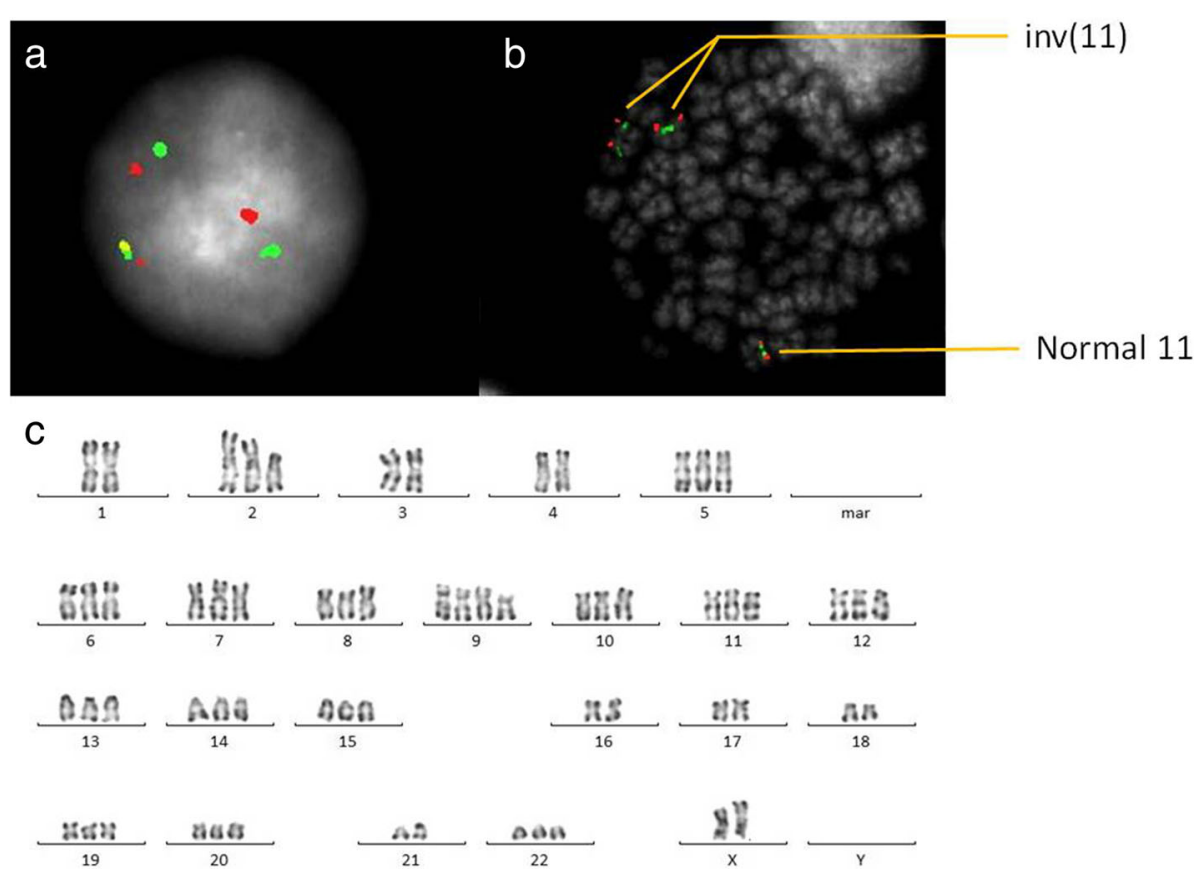

Fig. 1 Cytogenetic analysis at initial diagnosis. Ish inv. (11) (q21, 5'MLL+)(q23, 3'MLL+) ×2 [4].nucish (MIIx3, 5'MLLsep3'MLLx2) [58/100]. FISH study using a LSI KMT2A break apart probe (Vysis, Abbott Molecular Inc.): (a) an interphase cell showing one non-rearranged orange/green signal fusion and two centomeric (red) and two telomeric (green) separate signals, (b) a metaphase cell showing a normal chromosome 11 and two inverted chromosome 11, (c) karyotype from bone marrow aspirate showing complex chromosome abnormalities

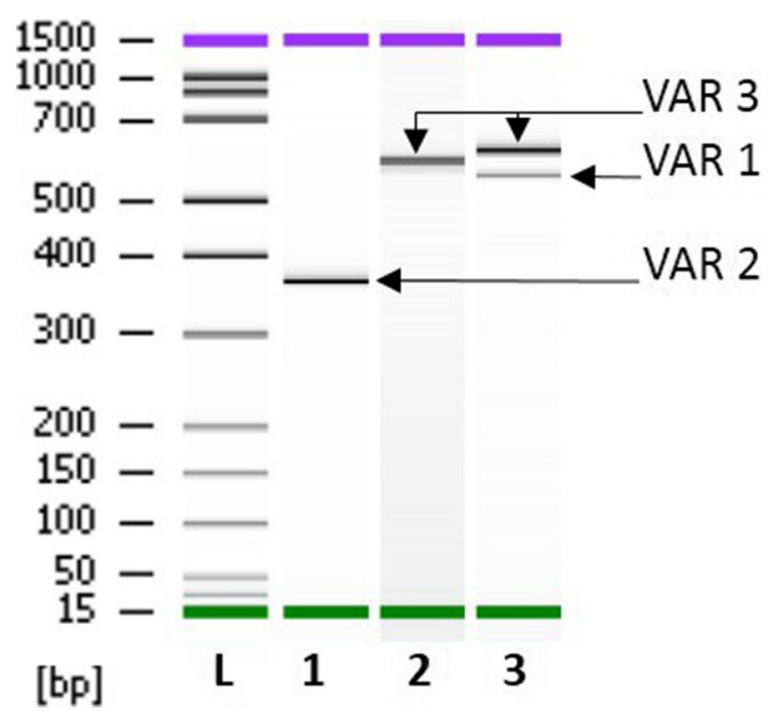

Fig. 2 RT-PCR analysis of KMT2A-MAML2 fusion gene. RT-PCR for the KMT2A-MAML2 fusion was performed from patient's RNA. $1 \mu \mathrm{g}$ total RNA were reverse-transcribed into cDNA in a $20 \mu$ total volume using random hexamer primers according to EAC protocol (Gabert et al.,2003). PCR reactions contained 1X PCR buffer (Applied Biosystems), $20 \mathrm{nmol}$ dNTPs (Applied Biosystems), $300 \mathrm{nM}$ primer of each primer, 1.25 units of AmpliTaq Gold polymerase (Applied Biosystems), and $5 \mu \mathrm{l}$ of CDNA in a 50- $\mu$ l reaction volume. Specific primers were used and were localized on exon 8 of KMT2A (5'-GTCCAGAGCAGAGCAAACAG-3') and intron 1-2 of MAML2 (5'-TCCCATCTCCAAGTCTCAGC-3') (Lane 1), on exon 8 of KMT2A and exon 2 of MAML2 (5'-GAGTCTCTCCTGGCTCCTTC-3') (Lane 2) and on exon 7 of KMT2A (5'-ATCCTGCCCCAAAGAAAAGC-3') and exon 2 of MAML2 (Lane 3). PCR products were analyzed with Agilent DNA 1000 kit using the 2100 bioanalyzer 
KMT2A

MAML2

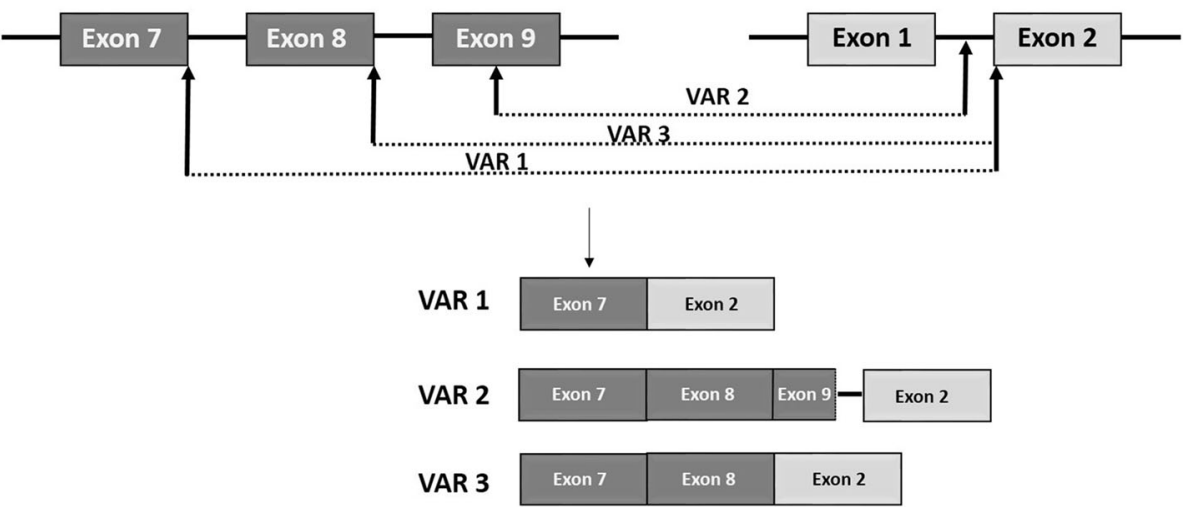

Fig. 3 KMT2A and MAML2 fusion transcripts. The KMT2A exon 7 and exon 8 were fused in-frame with MAML2 exon 2 corresponding to variant 1 (VAR1) and variant 3 (VAR3) respectively. The variant 2 (VAR2) was the resulting of a breakpoint within KMT2A exon 9 and within MAML2 intron $1-2$

Concerning the development of secondary leukaemia following chemo-radiotherapy for sarcoma, it is known that chemo-radiotherapy administered for a primary cancer will increase the risk of secondary neoplasms [10]. Alkylating agents have been reported as affecting the radiation therapy-associated risk for secondary malignancies [11]. However, in this case, the speed of development of a secondary leukemia is unusual. Alkylating agent/radiotherapy-related leukemia has a mean latency period of 5-7 years, are often preceded by a myelodysplastic phase and are frequently associated with complex karyotypes, whereas acute secondary leukaemia after treatment with DNA topoisomerase II inhibitors has a relatively short latency period (several months to 3 years) and is associated with chromosomal translocation involving $11 \mathrm{q} 23$, respectively the KMT2A gene $[12,13]$.

To conclude, clinical outcomes of $K M T 2 A$-associated leukemias are often unfavorable and identification of a $K M T 2 A$ partner could lead to new therapeutic strategies. Moreover, bringing to light several variants of a fusion transcript, such as the KMT2A-MAML2 gene, is important in the follow-up of residual disease.

\section{Abbreviations}

ALL: acute lymphoid leukemia; AML: acute myeloid leukemia;

FISH: fluorescent in situ hybridization;" MAML2: mastermind like 2;

MDS: myelodysplasic syndrom; NGS: Next-Generation Sequencing

\section{Acknowledgements}

We gratefully acknowledge technicians and clinicians for collaboration. The authors also like to acknowledge Drs Caroline Leonnet, Marie Loosveld and all the cytologists of La Timone Hospital laboratory (APHM) for their valuable assistance in this difficult diagnosis.

\section{Funding}

This work was not supported by any sources of funding.

\section{Availability of data and materials}

The sequence used in this study are publicly available from GenBank database (https://www.ncbi.nlm.nih.gov/genbank/) using the following accession numbers NM_001197104 (KMT2A mRNA) and NM_032427 (MAML2 $m R N A$ ). The authors declare that the data supporting the findings of this study are available within the article.

\section{Authors' contributions}

All cited authors qualify for authorship according to the ICMJE guidelines. EM, NB, FU, JG carried out the molecular studies. EB, MLP carried out the cytogenetic studies. RC carried out the clinical management of the patient. All authors read and approved the final manuscript.

\section{Competing interests}

The authors declare that they have no competing interests.

\section{Consent for publication}

Written informed consent was obtained from the patient for the publication of this report and any accompanying images.

\section{Ethics approval and consent to participate}

The AP-HM scientific committee approved the present study. In accordance with French regulations and due to the observational nature of this single patient retrospective study, no formal ethics approval is required.

\section{Publisher's Note}

Springer Nature remains neutral with regard to jurisdictional claims in published maps and institutional affiliations.

\section{Author details}

${ }^{1}$ Department of Biochemistry \& Molecular Biology, University Hospital Nord Marseille, France. 'U1072 INSERM, Université de la Méditerranée, Marseille, France. ${ }^{3}$ Genetic Department of AP-HM Marseille, Marseille, France.

${ }^{4}$ Department of clinical onco-hematology, University Hospital of La Conception, Marseille, France.

Received: 8 November 2016 Accepted: 17 May 2017

Published online: 23 May 2017

\section{References}

1. Yokoyama A. Molecular mechanisms of MLL-associated leukemia. Int J Hematol. 2015;101:352-61.

2. Saleem M, Yusoff NM. Fusion genes in malignant neoplastic disorders of haematopoietic system. Hematol Amst Neth. 2016;21:501-12.

3. Nemoto $N$, et al. Identification of a novel fusion gene MLL-MAML2 in secondary acute myelogenous leukemia and myelodysplastic syndrome with inv(11)(q21q23). Genes. Chromosomes Cancer. 2007;46:813-9.

4. Pagano $L$, et al. Acute lymphoblastic leukaemia occurring as second malignancy: report of the GIMEMA archive of adult acute leukaemia. 
Gruppo Italiano Malattie Ematologiche Maligne dell'Adulto. Br J Haematol. 1999;106:1037-40.

5. Metzler M, et al. Inv(11)(q21q23) fuses MLL to the Notch co-activator mastermind-like 2 in secondary T-cell acute lymphoblastic leukemia. Leukemia. 2008;22:1807-11.

6. Kumar S, Razzaq SK, Vo AD, Gautam M, Li H. Identifying fusion transcripts using next generation sequencing. Wiley Interdiscip Rev RNA. 2016. doi:10 1002/wrna.1382.

7. Scolnick JA, Dimon M, Wang I-C, Huelga SC, Amorese DA. An Efficient Method for Identifying Gene Fusions by Targeted RNA Sequencing from Fresh Frozen and FFPE Samples. PLoS One. 2015;10:e0128916.

8. Chen M, Manley JL. Mechanisms of alternative splicing regulation: insights from molecular and genomics approaches. Nat Rev Mol Cell Biol. 2009;10: $741-54$

9. Daber R, Sukhadia S, Morrissette JJD. Understanding the limitations of next generation sequencing informatics, an approach to clinical pipeline validation using artificial data sets. Cancer Genet. 2013;206:441-8.

10. Leone G, Mele L, Pulsoni A, Equitani F, Pagano L. The incidence of secondary leukemias. Haematologica. 1999;84:937-45.

11. Goudarzi Pour K, et al. Secondary ALL after Successful Treatment of Ewing's Sarcoma: A Case Report. Int J Hematol-Oncol Stem Cell Res. 2016;10:236-8.

12. Douet-Guilbert N, et al. MLL partner genes in secondary acute lymphoblastic leukemia: report of a new partner PRRC1 and review of the literature. Leuk Res. 2014;38:1316-9.

13. Rowley JD, Olney HJ. International workshop on the relationship of prior therapy to balanced chromosome aberrations in therapy-related myelodysplastic syndromes and acute leukemia: overview report. Genes Chromosomes Cancer. 2002;33:331-45.

\section{Submit your next manuscript to BioMed Central and we will help you at every step:}

- We accept pre-submission inquiries

- Our selector tool helps you to find the most relevant journal

- We provide round the clock customer support

- Convenient online submission

- Thorough peer review

- Inclusion in PubMed and all major indexing services

- Maximum visibility for your research

Submit your manuscript at www.biomedcentral.com/submit

) Biomed Central 